\title{
Celiac Disease and Maternal Infertility and Pregnancy Outcomes: Is Screening Necessary?
}

\author{
Syed Mohammad ${ }^{1}$, Kai $\mathrm{Wu}^{1}$, Johnny Huang ${ }^{1}$ \\ ${ }^{1}$ Faculty of Medicine, University of Ottawa
}

\section{ABSTRACT}

Celiac disease is a common autoimmune condition that is often underappreciated in pregnant women. Due to the difficulty in conducting high-quality studies involving pregnant patients, the evidence supporting the association between celiac disease and maternal fertility and pregnancy outcome, and the benefits of screening for celiac disease in this population are unclear. Therefore, we sought to review the relevant literature to gain a better understanding of the impact of celiac disease on maternal fertility and fetal outcome. Our findings suggest a role for celiac screening in women with unexplained infertility.

\section{RÉSUMÉ}

La maladie cœliaque est une maladie auto-immune commune qui est souvent sous-estimée chez les femmes enceintes. En raison de la difficulté à mener des études de haute qualité impliquant des patientes enceintes, les preuves soutenant l'association entre la maladie cœliaque et la fertilité maternelle et l'issue de la grossesse, et les avantages du dépistage de la maladie cœliaque dans cette population ne sont pas claires. Par conséquent, nous avons cherché à examiner la littérature pertinente pour mieux comprendre l'impact de la maladie cœeliaque sur la fertilité maternelle et l'issue fœtale. Nos résultats suggèrent un rôle pour le dépistage de la maladie cœliaque chez les femmes présentant une infertilité inexpliquée.

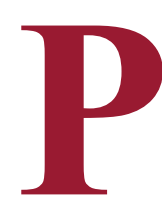

regnancy provides unique challenges to healthcare providers. A plethora of questions remain regarding the effects of investigations, management options, and conditions on pregnancy. Incomplete knowledge of these aspects stems from the difficulty in conducting high-quality studies involving pregnancy because of the many patient, ethical, and legal factors involved in researching this vulnerable group consisting of the mother and the fetus [1].

Celiac disease is more common in females and its effect on pregnancy and fetal outcome is controversial [2]. In celiac disease, the immune system inappropriately reacts to gluten in the small intestine, which results in varying degrees of small bowel damage. It can be diagnosed using a combination of serological testing for autoantibodies and small bowel biopsy, depending on the clinical index of suspicion for the disease. By going on a gluten free diet, patients can prevent the inflammation and subsequent damage that occurs in the small intestine. Previous studies have hinted at a possible increased prevalence of celiac disease in pregnant women. For example, a study by Martinelli et al. found that $1.4 \%$ of a study population of 845 pregnant women had previously undiagnosed celiac disease [3], which was comparable to the prevalence of other routinely screened conditions [4]. They found that there was in turn a statistically significant increase in adverse pregnancy outcomes in this population. Based on the study results, the authors recommended routine testing of celiac disease during pregnancy. Despite these studies, there is insufficient evidence supporting the association between celiac disease and maternal fertility and pregnancy outcome. The benefits of screening for celiac disease in this population remain unclear. Therefore, we sought to review the relevant literature to gain a better understanding of the impact of celiac disease on maternal fertility and fetal outcome, and based on this evaluate the utility of routine screening of celiac disease in pregnancy.

\section{PROPOSED IMPACTS OF CELIAC DISEASE ON PREGNANCY}

Celiac disease has been postulated to affect maternal fertility and pregnancy outcomes through two mechanisms: nutritional deficiency and autoimmune dysregulation [4]. Celiac disease can result in the malabsorption of zinc, selenium, and folic acid, which are essential compounds for pregnancy [4,5]. The autoimmune hypothesis postulates that either anti-transglutaminase (tTG) antibodies bind to the trophoblast layer of the embryo, causing damage to the future placenta, or that anti-tTG antibodies can harm the maternal endometrial endothelial cells 
$[4,5]$. Studies have shown that women who suffer from infertility associated with villous atrophy caused by celiac disease do not have signs of absorption deficiency $[6,7]$. This suggests that celiac disease affects pregnancy through an autoimmune process that affects the placenta before and during the pregnancy, rather than through inducing nutritional deficiency. Celiac disease is frequently asymptomatic for long periods while still having histological effects, so it is possible undiagnosed celiac disease may impact pregnancy while being clinically undetected.

\section{INFERTILITY}

Infertility, defined as the inability to conceive for 1 year, can have devastating impacts on mental health [8]. In $15-30 \%$ of infertility cases, no cause can be found [9]. An often undiagnosed disease, celiac disease has been postulated to influence fertility and could explain a portion of infertility cases.

A study by Collin et al. from 1996 compared 150 controls with 98 women with infertility of unknown origin [10]. Of the 98 , four had previously undiagnosed celiac disease that was diagnosed by serological testing for autoantibodies, compared to none from the control group, a statistically significant difference. Another study conducted by Meloni et al. from 1999 demonstrated similar results showing 4 out of 99 women with unexplained infertility having positive serological markers for celiac disease, and 3 of those 4 having histological evidence, a statistically significant difference compared to the prevalence of celiac disease in the general population (0.5-1\%) [7].

There were two recent studies by Shamaly et al. and Tiboni et al. on 192 women and 200 women, respectively. Although they both found an association between previously undiagnosed celiac disease and infertility, neither study reached statistical significance with their outcome $[6,11]$. Shamaly et al's study had 4 patients with celiac disease in their infertility group with celiac compared to one in the control group, and Tiboni et al. found 5 and 2 , respectively $[6,11]$. The authors of both papers attributed the lack of statistical significance due to the studies being underpowered. On the surface, it may seem that both had a large sample size, but given the very low prevalence of celiac disease $(0.5 \%)$, even reasonably large sample sizes will not yield many celiac cases, making comparisons difficult. It is therefore not possible to make strong conclusions based on the data currently available.

There is very limited data present that suggests the possibility that a gluten free diet may improve fertility outcomes in women with celiac disease. A study performed by Sher \& Mayberry utilized questionnaires to survey 80 patients with undiagnosed celiac disease and 70 age and sex-matched controls, and found that women with celiac disease had statistically significant less children overall (120) compared to the control group (161). Using the questionnaires they found women with celiac disease had fewer children overall (120) compared to controls - a finding that was statistically significant.Furthermore, a case report by Rajput and Shatterjee outline the case of an infertile woman found to have infertility which was successfully treated using a gluten free diet $[12,13]$.

Clearly, the data regarding celiac disease and infertility, as well as the possible effect of a gluten free diet, is highly limited. However, given the presence of an association in all of the studies, when faced with infertility unexplained by other factors, we feel it is reasonable to consider a workup for celiac disease in this population as a possible contributing factor to infertility. A screening test simply requires looking for serological presence of autoantibodies and so is straightforward, and yet could potentially help explain some infertility cases. This may be especially important as there are very small amounts of data suggesting the possibility that treatment of celiac disease may lead to subsequent fertility improvement.

\section{FETAL OUTCOME}

Several retrospective studies have examined the potential impact of undiagnosed celiac disease on fetal outcome [3,14-16]. All of them examined parents of children with poor birth outcomes. Among these, a noteworthy retrospective study by Salvatore et al. investigated 1,714 parents ( 868 women, 846 men) of preterm and/or small for gestational age (SGA) infants for celiac disease [14]. The study found previously undiagnosed celiac disease to be a risk factor for these adverse outcomes. However, despite the very large sample size, the absolute number of subjects with celiac disease in this study was still low, and thereby precluded further subgroup analysis. Despite the limitations of sample size, the trend among all of these retrospective studies suggests that celiac disease is associated with adverse fetal outcomes such as SGA, preterm, low birth weight (LBW), miscarriage, and intrauterine growth restriction (IUGR). When simply basing off of the collection of evidence in these relatively small studies, it would appear that there is some evidence to suggest celiac disease may have a link with adverse birth outcomes. 
However, more recent population-based studies with very large sample sizes cloud the picture. Two large retrospective cohort, population-based, studies using the United Kingdom (UK) population, one involving over 2.5 million women, did not demonstrate an association between undiagnosed and diagnosed celiac disease and adverse pregnancy and birth outcomes $[17,18]$. Conversely, a population study of the Danish population with a sample size over 1.5 million demonstrated that untreated celiac disease could lead to poor fetal outcomes, but presumed treatment (as defined by those with celiac diagnosis prior to 90 days before the start of pregnancy) led to no discernible difference [19].

Clearly, the assumption regarding the definition of the treated group is a large one, but nonetheless the difference in the results for the two study groups in the Danish study may help to explain the discrepancy between the results of the UK population-based studies and the smaller retrospective studies mentioned earlier. It is possible that the difference in results between the studies may be due to differences in study designs. The non-population-based studies performed retrospective analyses to correlate the presence of parental celiac disease and poor offspring outcome. Simply put, they examined the parents of children who have already had an adverse birth outcome (i.e. SGA), and then worked back in order to see if celiac disease was more common in these parents (i.e. look at effect, and work back to find the cause). This form of study design meant the researchers were only capturing previously undiagnosed celiac disease. On the other hand, the population studies track a large number of individuals with celiac disease, and then look at what happened to all of their children (i.e. look at a potential cause, and go forward to see the outcome). This form of study design would capture outcomes of patients with known celiac disease, which the other studies did not. We theorize, therefore, that the retrospective studies are only capturing a subset of celiac patients that may have a specific feature that put them at higher risk (i.e. they were all undiagnosed celiac disease), but this risk does not translate across celiac patients in general who may be receiving treatment.

Furthermore, this theory also follows the autoimmune mechanism mentioned earlier-anti-tTG antibodies are typically found in patients with active celiac disease which would explain why a gluten free diet eliminates most of the pregnancy complications found by the researchers. Taken in combination with our postulated theory, it would certainly appear that a fac- tor such as disease severity may play a role in the relationship between celiac and pregnancy, and could very well explain the inconsistency between the population-based studies and the retrospective ones.

\section{CELIAC DISEASE SCREENING}

Given that, on the population level, celiac disease has not been shown to be associated with adverse birth outcomes, we do not recommend the universal screening of pregnant women for celiac disease. Even though some retrospective studies showed a link between poor birth outcome and celiac disease, this finding may only be associated with a subset of previously undiagnosed celiac disease patients, which is a very small group. This recommendation is supported by a study by Greco et al. from 2004 which demonstrated that screening of 5,055 pregnant women near delivery for celiac did not result in prevention of adverse fetal outcome [20]. However, they only screened with antibodies, and never confirmed the diagnosis with a tissue biopsy. On the basis of the aforementioned evidence, the benefits of screening for celiac disease in pregnant women does not appear to be clinically significant.

\section{CONCLUSION}

Celiac disease is a common autoimmune condition that is an often underappreciated disease in pregnant women. Increased awareness and adherence to an appropriate gluten free diet should be promoted among this population to mitigate infertility rates and improve reproductive health. Healthcare providers may consider investigating women with unexplained infertility. However, more studies are needed to evaluate the utility of universal screening for celiac disease in pregnant women.

\section{REFERENCES}

1. Naqvi TZ. Challenges in cardiology research in pregnancy. Future Cardiol. 2014;10(6):759-68.

2. Ciacci C, Cirillo M, Sollazzo R, Savino G, Sabbatini F, Mazzacca G. Gender and clinical presentation in adult celiac disease. Scand J Gastroenterol. 1995;30(11):1077-81.

3. Martinelli $\mathrm{P}$, Troncone R, Paparo F, et al. Coeliac disease and unfavourable outcome of pregnancy. Gut. 2000;46(3):332-5.

4. Tersigni $C$, Castellani R, De Waure $C$, et al. Celiac disease and reproductive disorders: meta-analysis of epidemiologic associations and potential pathogenic mechanisms. Hum Reprod Update. 2014;20(4):582-93.

5. Robinson NJ, Glazier JD, Greenwood SL, Baker PN, Aplin JD. Tissue transglutaminase expression and activity in placenta. Placenta. 2006;27(2):148-57.

6. Shamaly H, Mahameed A, Sharony A, Shamir R. Infertility and celiac disease: do we need more than one serological marker? Acta Obstet Gynecol Scand. 2004;83(12):1184-8.

7. Meloni GF, Dessole S, Vargiu N, Tomasi PA, Musumeci S. The prevalence of coeliac disease in infertility. Hum Reprod. 1999;14(11):2759-61.

8. Galhardo A, Cunha M, Pinto-Gouveia J. Psychological aspects in couples with infertility. Sexologies. 2011;20(4):224-8. 
9. Quaas A, Dokras A. Diagnosis and treatment of unexplained infertility. Rev Obstet Gynecol. 2008;1(2):69.

10. Collin P, Vilska S, Heinonen PK, Hällström $O$, Pikkarainen P. Infertility and coeliac disease. Gut. 1996;39(3):382-4.

11. Tiboni GM, de Vita MG, Faricelli R, Giampietro F, Liberati M. Serological testing for celiac disease in women undergoing assisted reproduction techniques. Hum Reprod. 2005;21(2):376-9.

12. Rajput $R$, Chatterjee $S$. Primary infertility as a rare presentation of celiac disease. Fertil Steril. 2010;94(7):2771-e5.

13. Sher KS, Mayberry JF. Female fertility, obstetric and gynaecological history in coeliac disease: a case control study. Acta Pædiatrica. 1996;85(s412):76-7.

14. Salvatore S, Finazzi S, Radaelli G, Lotzniker M, Zuccotti GV. Prevalence of undiagnosed celiac disease in the parents of preterm and/or small for gestational age infants. Am J Gastroenterol. 2007;102(1):168.

15. Gasbarrini A, Torre ES, Trivellini C, De Carolis S, Caruso A, Gasbarrini G. Recurrent spontaneous abortion and intrauterine fetal growth retardation as symptoms of coeliac disease. Lancet. 2000;356(9227):399-400.

16. Moleski SM, Lindenmeyer CC, Veloski JJ, et al. Increased rates of pregnancy complications in women with celiac disease. Ann Gastroenterol Q Publ Hell Soc Gastroenterol. 2015;28(2):236.

17. Dhalwani NN, West J, Sultan AA, Ban L, Tata LJ. Women with celiac disease present with fertility problems no more often than women in the general population. Gastroenterology. 2014;147(6):1267-74.

18. Sultan AA, Tata LJ, Fleming KM, et al. Pregnancy complications and adverse birth outcomes among women with celiac disease: a population-based study from England. Am J Gastroenterol. 2014;109(10):1653.

19. Khashan AS, Henriksen TB, Mortensen PB, et al. The impact of maternal celiac disease on birthweight and preterm birth: a Danish population-based cohort study. Hum Reprod. 2009;25(2):528-34.

20. Greco L, Veneziano A, Di Donato L, et al. Undiagnosed coeliac disease does not appear to be associated with unfavourable outcome of pregnancy. Gut. 2004;53(1):149-51. 